\title{
Efecto Protector de un Antimicrobiano Natural Frente a Listeria monocytogenes, Salmonella Typhimurium y E. coli en Salchicha y Mortadela
}

\author{
Andrea Zapata-Álvarez ${ }^{1 *}$, Carlos E. Mejía ${ }^{2}$, Diego A. Restrepo-Molina ${ }^{3}$ \\ (1) Universidad de Antioquia, Sede Medellín. Facultad de Ciencias Exactas y Naturales. Instituto de \\ Biología. Grupo de Agrobiotecnología. Calle 67 No. 53 - 108. Medellín, Colombia. \\ (e-mail: andrea.zapata1@udea.edu.co) \\ (2) Universidad de Antioquia, Sede Medellín. Escuela de Microbiología. Grupo de Biotransformaciones. \\ Calle 67 No. 53 - 108. Medellín, Colombia. (e-mail: mcemg@udea.edu.co) \\ (3) Universidad Nacional de Colombia, Sede Medellín. Departamento de Ingeniería Agrícola y Alimentos. \\ Calle 59르 \# 63-20Medellín, Colombia. (e-mail: darestre@unal.edu.co) \\ ${ }^{*}$ Autor a quien debe ser dirigida la correspondencia
}

Recibido Ago. 7, 2018; Aceptado Oct. 24, 2018; Versión final Dic. 1, 2018, Publicado Abr. 2019

\begin{abstract}
Resumen
En este trabajo se evaluó el crecimiento de Listeria monocytogenes, Salmonella Typhimurium y E. coli $\left(10^{2}\right.$ $\left.10^{3} \mathrm{UFC} / \mathrm{ml}\right)$ en productos cárnicos formulados con un antimicrobiano natural $(0,3 \%$ y $0,65 \%)$ que contiene extracto de romero. E. coli fue inoculada en salchichas, mientras que L. monocytogenes y S. Typhimurium se inocularon por separado en mortadelas. Los productos se empacaron al vacío y almacenaron bajo refrigeración $\left(4-5^{\circ} \mathrm{C}\right)$. El antimicrobiano natural a las dosis estudiadas reduce más del $50 \%$ la población de E. coli y L. monocytogenes desde la segunda semana, e inhibe el $100 \%$ de la población a la dosis más alta para la segunda y tercera semana, respectivamente. Sólo se logró inhibir completamente a S. Typhimurium al final del estudio en los productos con la concentración más alta de antimicrobianos. Basado en los resultados del estudio, se concluye que el antimicrobiano natural inhibe el crecimiento de microorganismos patógenos, principalmente E. coli y L. monocytogenes.
\end{abstract}

\section{Protective Effect of a Natural Antimicrobial Against Listeria monocytogenes, Salmonella Typhimurium and E. coli in Salchicha y Mortadela}

\begin{abstract}
In this work the growth of Listeria monocytogenes, Salmonella Typhimurium and E. coli $\left(10^{2}-10^{3} \mathrm{CFU} / \mathrm{mL}\right)$ in meat products formulated with a natural antimicrobial $(0.3 \%$ and $0.65 \%)$ containing rosemary extract was evaluated. E. coli was inoculated into sausages, while L. monocytogenes and S. Typhimurium were inoculated separately into mortadella. The products were vacuum packed and stored under refrigeration $\left(4-5^{\circ} \mathrm{C}\right)$. The natural antimicrobial at the doses studied reduces more than $50 \%$ the population of $E$. coli and $L$. monocytogenes from the second week, and inhibits $100 \%$ of the population at the highest dose for the second and third week, respectively. Only S. Typhimurium was completely inhibited at the end of the study in the products with the highest concentration of antimicrobials. Based on the results of the study, it is concluded that the natural antimicrobial inhibits the growth of pathogenic microorganisms, mainly $E$. coli and $L$. monocytogenes.
\end{abstract}

Keywords: Listeria monocytogenes; Salmonella Typhimurium; E. coli; extracto de romero 


\section{INTRODUCCIÒN}

Salmonella Typhimurium, Listeria monocytogenes, y E. coli, hacen parte de la lista de las 10 principales bacterias causantes de la mayoría de los casos confirmados de infecciones alimentarias en EEUU (www.cdc.gov, 2010). En Colombia, el Instituto Nacional de Vigilancia de Alimentos y Medicamentos, INVIMA (www.invima.gov.co, 2017), indicó que en el país ocurren cerca de 240.000 casos de infecciones transmitidas por alimentos al año. Debido a un subregistro en el número de casos notificados, es difícil conocer las cifras de los brotes ocasionados por el consumo de productos cárnicos procesados, y mucho más, identificar el agente causal. Sin embargo, se sabe que por sus características intrínsecas, los productos y derivados cárnicos como salchicha y mortadela, se encuentran entre los alimentos con mayor riesgo de ocasionar una infección causada por bacterias como Listeria monocytogenes, Salmonella Typhimurium, E. coli.

Los productores además de adoptar adecuadas prácticas de manufactura y un correcto plan de limpieza y desinfección en sus plantas, deben asegurar también la inocuidad de los productos durante su almacenamiento y distribución. Una de las prácticas más empleadas para lograr este objetivo, es la adición de agentes antimicrobianos en las fórmulas cárnicas como el lactato sódico, que además de ser considerado GRAS (Generally Recognized As Safe), se recomienda como parte del programa para el control de $L$. monocytogenes en alimentos listos para su consumo (ready-to-eat, RTE) (Ahmed et al., 2015) y para inhibir el crecimiento de microorganismos mesófilos y Clostridium sporogenes (Pereira et al., 2015). El efecto bacteriostático de los lactatos se debe al aumento de la fase de latencia de los microorganismos, y su mecanismo de inhibición ha sido atribuido a la facilidad de estos ácidos lipofílicos débiles para ingresar a través de la membrana celular en su forma no disociada, y una vez dentro, disociarse y acidificar el medio intracelular (Mbandi y Shelef, 2001). Sin embargo, pese a la efectividad antimicrobiana del lactato de sodio, las concentraciones en las que se observan los mejores resultados (1-3\%) suelen ser muy elevadas comparadas con otros aditivos, y su aporte de sodio es por consiguiente, muy alto. Es por tal motivo que se han venido empleando otra clase de antimicrobianos para remplazarlo.

En los últimos años, ha habido un creciente interés por el uso de antimicrobianos naturales (Pisoschi et al., 2018). Los aceites esenciales de la mayoría de las plantas son ricos en fenoles de reconocida actividad antimicrobiana como carvacrol, eugenol y timol que ayudan a aumentar la vida útil de una gran variedad de alimentos (Muriel et al., 2015), debido a que alteran la bicapa de fosfolípidos de la membrana celular y afectan negativamente los sistemas enzimáticos y el material genético de los microorganismos (Bezic et al., 2013). El romero (Rosmarinus officinalis I.) se utiliza exitosamente en la industria de los alimentos por su actividad antioxidante, y como agente antimicrobiano se ha estudiado su acción frente bacterias Gram-negativas como Escherichia coli 0157:H7 y Aeromonas hydrophila; además de Gram-positivas como Listeria monocytogenes (Ghabraie et al., 2016). El extracto de romero muestra individualmente una fuerte actividad antimicrobiana en carne de cerdo fresca y en rodajas de jamón envasado al vacío y con atmósferas modificadas, frente a Escherichia coli, Pseudomonas fluorescens y Lactobacillus sake (Kalschne, D., et al., 2015). Se ha demostraron in vitro que independiente de su naturaleza lipofílica o hidrofílica, el extracto de romero inhibe $L$. monocytogenes y una gran variedad de bacterias acidolácticas (Bubonja et al., 2011).

Con el objetivo de evaluar el crecimiento de Listeria monocytogenes, Salmonella Typhimurium y E. coli en salchichas y mortadelas formuladas con un antimicrobiano natural que contiene extracto de romero, se diseñó una prueba de desafío. El seguimiento microbiológico para obtener el recuento de cada microrganismo se realizó semanalmente durante la vida útil comercial del producto.

\section{MATERIALES Y MÉTODOS}

Se elaboraron salchichas y mortadelas mediante metodología tradicional, según se presenta en la Figura 1. Las mortadelas se inocularon por separado con Listeria monocytogenes y Salmonella Typhimurium, mientras que Escherichia coli se inoculó en salchicha. Semanalmente se realizó un seguimiento microbiológico de los productos que sirvió para comparar la eficacia de los diferentes antimicrobianos.

\section{Cepas bacterianas}

La cepa de Listeria monocytogenes $\left(\right.$ ATCC $^{\oplus} 13932^{\mathrm{TM}}$ ), Escherichia coli $\left(\right.$ ATCC $\left.^{\circledR} 25922^{\mathrm{TM}}\right)$ ) y Salmonella enterica subsp. entericaserovar Typhimurium (ATCC ${ }^{\circledR} 14028^{\text {TM}}$ ) empleadas en este estudio se almacenaron, cultivaron y analizaron siguiendo las indicaciones del proveedor (Laboratorios Annar, Bogotà).

\section{Formulación y elaboración de los productos}

Para la elaboración de las salchichas y mortadelas se empleó carne bovina, pasta /piel pollo y pasta de pollo (Tyson, México) comprada a un proveedor local. Los demás ingredientes los proporcionó Tecnas S.A., Itagüí, 
Colombia. Estos son: sabor natural pollo (mezcla de especias y extractos), almidón de papa (Aris ${ }^{\circledR}$, Mèxico), sal (Refisal ${ }^{\circledR}$, Colombia), proteína vegetal hidrolizada (Levapan ${ }^{\circledR}$, Bogotá, Colombia), proteína aislada y texturizada de soya (Solae, LLC, St. Louis, Missouri, Estados Unidos), eritorbato de sodio (C6H7NaO6, Jebsen \& JessenG.m.b.H. \& Co.), tripolifosfato de sodio ( $\mathrm{Na}_{5} \mathrm{P}_{3} \mathrm{O}_{10}$, Haifa Chemicals, Haifa, Israel) grado alimenticio, nitrito de sodio (6\% nitrito de sodio, $\mathrm{NaNO}_{2}, 94 \% \mathrm{NaCl}$ ), colorante natural para embutidos (Color $\mathrm{Ful}^{\circledR}$, Tecnas, Colombia), extracto de romero (Kalsec ${ }^{\circledR}$, Estados Unidos) con el que se prepara el antimicrobiano natural y lactato de sodio (Galactic ${ }^{\circledR}$, Estados Unidos) este último usado como antimicrobiano de referencia. La concentración de los principales componentes de la fórmula se muestra en la Tabla 1 . Todas las fórmulas se balancearon garantizando el mismo contenido de humedad y sal en los tratamientos.

Previamente, usando el método de dilución en caldo se determinó la Concentración Mínima Inhibitoria (CMI) en la cual el lactato de sodio (control positivo) y el antimicrobiano natural compuesto principalmente por extracto de romero inhiben todas las cepas de interés. En este estudio se evaluó la efectividad de un antimicrobiano natural a dos concentraciones $(0,3 \%$ y $0,65 \%)$ y se comparó frente al control sin ningún tipo de agente microbiano en salchicha inoculada con $E$. coli, y mortadelas inoculadas por separado con $L$. monocytogenes y $S$. Typhimurium, empacadas al vacío y almacenadas a temperatura de refrigeración (4-5 $\left.{ }^{\circ} \mathrm{C}\right)$. Semanalmente, se determinó el recuento de estas bacterias y se realizaron pruebas microbiológicas de rutina y detección de L. monocytogenes y Salmonella spp. en los productos que no habían sido inoculados.

Tabla 1: Composición de los principales ingredientes de la salchicha y mortadela.

\begin{tabular}{|c|c|}
\hline Ingrediente & Composición (\%) \\
\hline Res (bovino) & 12 \\
\hline Pasta de pollo & 46 \\
\hline Tocino de cerdo/piel de pollo & 13 \\
\hline Agua/Hielo & 14.6 (aprox.) \\
\hline Almidón de papa & 5,8 \\
\hline Proteína de soya & 3,0 \\
\hline Sal & 1,1 \\
\hline Proteína vegetal hidrolizada & 0,5 \\
\hline Polifosfato de sodio & 0,4 \\
\hline Eritorbato de sodio & 0,05 \\
\hline Nitrito de sodio & 0,02 \\
\hline
\end{tabular}

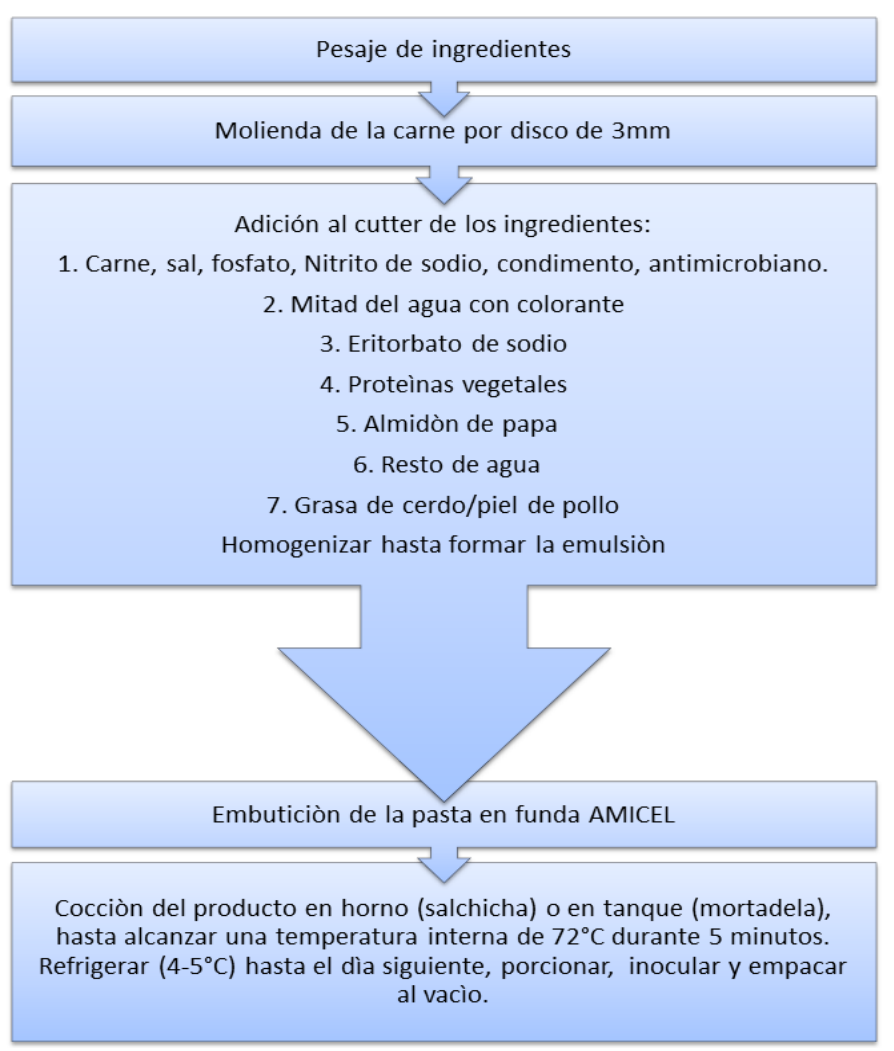

Fig. 1: Elaboración de salchicha y mortadela. 
Para reducir el tamaño del material cárnico se empleó un molino de discos M22 R2 (C.I. Talsa, Medellin, Colombia). El material cárnico se dividió en 4 porciones iguales, y se mantuvo durante 24 horas en refrigeración $\left(4^{\circ} \mathrm{C}\right)$ para su uso posterior. Transcurrido este tiempo, todos los ingredientes se adicionaron a un Cutter Cruells de 15 litros de capacidad (C.I. Talsa, Medellin, Colombia) en el orden que se muestra en la Figura 1. La pasta se embutió usando una embutidora hidráulica Mainca EM-30 (C.I. Talsa, Medellin, Colombia), en funda de Amicel (película coextruida de 70 micras, poliamida/adhesivo/capa sellante, PEBD) proporcionada por la empresa Alico S.A. La masa así embutida se porcionó usando una amarradora manual (C.I. Talsa, Medellin, Colombia), y luego se llevó a cocción en un horno automático C.I. Talsa r100 1 carro (salchicha) o tanque de cocción a gas C.I. Talsa t240g - 09401085 (mortadela) hasta que el producto alcanzó una temperatura interna de $72^{\circ} \mathrm{C}$; después del tratamiento térmico, las salchichas y las mortadelas se dejaron en refrigeración y al siguiente día se realizó la inoculación del producto.

\section{Inoculación de los productos}

A partir de un inóculo que estaba en medio BHI (Brain Heart Infusion) con una concentración inicial de $10^{8}$ UFC/mL, se realizaron diluciones seriadas hasta tener una concentración aproximada de $10^{2}-10^{3} \mathrm{UFC} / \mathrm{mL}$ de cada cepa de referencia. Dentro de cada paquete, las salchichas se inyectaron con $1 \mathrm{~mL}$ de este último inóculo, mientras que en la superficie de las mortadelas se realizó la aspersión de $1 \mathrm{~mL}$ de $L$. monocytogenes o S. Typhimurium. Cada uno de los tratamientos se realizó para simular las condiciones reales en las que ocurre una re-contaminación con L. monocytogenes y S. Typhimurium después del tratamiento térmico de los productos, durante el lonchado (Ahmed et al., 2015) o, la presencia de E. coli proveniente de materias primas de calidad microbiológica deficiente. Una vez inoculados los productos, los paquetes se empacaron al vacío en una bolsa flexible de poliamida/polietileno (Alico S.A., Medellin, Colombia) usando la empacadora Cunet Plus Vac 20 (C.I. Talsa, Medellin, Colombia). El producto se mantuvo en refrigeración (4-5 $\left.{ }^{\circ} \mathrm{C}\right)$ en una Cava LaSelle de 500L durante el tiempo que duró el estudio, retirando cada muestra 1-2 horas antes de ser analizadas.

\section{Análisis microbiológico}

El análisis microbiológicos de las unidades sin inocular se llevó a cabo de acuerdo con las Normas Técnicas Colombianas, NTC 4519 para aerobios mesófilos (Instituto Colombiano de Normas Técnicas y Certificación, ICONTEC, 2009a), NTC 4516 para recuento de coliformes (ICONTEC, 2009b), y al manual de técnicas de análisis para control de calidad microbiológica de alimentos para consumo (Staphylococcus aureus, y Salmonella spp.). El recuento de L. monocytogenes y S. Typhimurium se realizó de acuerdo con las normas de la Organización Internacional de Normalización, ISO: ISO 11290-2: 01/07/1998 e ISO 6579:2003, respectivamente.

El recuento de L. monocytogenes, S. Typhimurium y E. coli de los productos inoculados, se realizó semanalmente como se describe a continuación. Cada vez se emplearon $25 \mathrm{~g}$ de muestra inoculada con $S$. Typhimurium homogeneizada en $225 \mathrm{~mL}$ de agua peptona tamponada, los cuales se incubaron a $36{ }^{\circ} \mathrm{C} \pm 2$ ○C por 18-24 horas. Para el enriquecimiento selectivo se transfirieron $1 \mathrm{~mL}$ de la muestra anterior en $10 \mathrm{~mL}$ de caldo Rappaport Vassiliadis y $10 \mathrm{~mL}$ de caldo Selenito cisteína, los cuales se incubaron a $44{ }^{\circ} \mathrm{C} \pm 2{ }^{\circ} \mathrm{C}$ y $36^{\circ} \mathrm{C} \pm 2{ }^{\circ} \mathrm{C}$ durante $18-24$ horas, respectivamente. El aislamiento en medio selectivo y diferencial se realizó a partir de cada uno de los cultivos obtenidos del enriquecimiento selectivo, los cuales se sembraron en superficie sobre el Agar SS (Agar Salmonella-Shigella), luego de incubar las placas a $36{ }^{\circ} \mathrm{C} \pm 2{ }^{\circ} \mathrm{C}$ durante 24-48 horas.

El recuento en placa de E. coli se consiguió en un agar cromogénico Chromocult ${ }^{\circledR}$ sobre el cual se sembró en superficie $0,1 \mathrm{~mL}$ de las diluciones correspondientes. Las cajas de Petri se incubaron a $35^{\circ} \mathrm{C} \pm 2{ }^{\circ} \mathrm{C}$ durante 24 horas para coliformes totales y para determinación de $E$. coli se incubaron a $44^{\circ} \mathrm{C} \pm 1{ }^{\circ} \mathrm{C}$ por $18-20$ horas. En el caso de L. monocytogenes, luego del proceso de recuperación del microorganismo en caldo Fraser siguiendo las indicaciones del proveedor, el recuento en placa se realizó por inoculación de $0,1 \mathrm{~mL}$ de la muestra en un medio selectivo diferencial ALOA incubando las placas a $36{ }^{\circ} \mathrm{C} \pm 2{ }^{\circ} \mathrm{C}$ durante 24 horas. Después del periodo de incubación de los microorganismos en las placas, se cuentan las colonias características en cada caja y se calculan el número de Unidades Formadoras de Colonias (UFC) por gramo.

\section{Análisis fisicoquímico}

Los valores de $\mathrm{pH}$ y actividad acuosa $(\mathrm{Aw})$ de las salchichas y mortadelas cocidas sin inocular se midieron semanalmente por medio de un pHmetro handylab pH11/14 (SchottGroup, Mainz, Germany) y un equipo AquaLab Water Activity Meter 4TE (AquaLab Inc., Pullman, WA 99163 - USA), respectivamente, según las instrucciones del fabricante. En ambos casos, se tomaron los promedios de 6 mediciones realizadas a 3 muestras de cada tratamiento, las cuales primero se maceraron hasta obtener una pasta homogénea. 


\section{Análisis estadístico}

Los recuentos de cada microorganismo se transformaron a logaritmo y se reportaron en términos de Log UFC/g. Cuando no se observó crecimiento en la placa, se utilizó el valor de 1 para el cálculo de su logaritmo. Los análisis se realizaron utilizando el Sotware estadístico $R\left(R C o r e T e a m^{\circledR}, 2012\right)$, a través del cual se obtuvo el modelo matemático de efectos mixtos (medidas longitudinales o repetidas en el tiempo) para cada microorganismo y tratamiento antimicrobiano (Littell, R., et al., 2006). El modelo con el mejor ajuste de los datos se determinó de acuerdo con el criterio de Akaike (AIC) (Posada S. y Rosero, R; 2007).

\section{RESULTADOS}

A continuación se presentan los resultados de los parámetros microbiológicos y fisicoquímicos de los productos no-inoculados. Además, se muestra la cinética de crecimiento de $E$. coli, L. monocytogenes y $S$. Typhimurium en los productos que se inocularon $\left(10^{2}-10^{3} \mathrm{UFC} / \mathrm{mL}\right)$, y el porcentaje de inhibición de cada microorganismo comparando el crecimiento sin ninguna sustancia antimicrobiana (Control) y el obtenido con el antimicrobiano en cuestión.

\section{Análisis microbiológico y fisicoquímico}

En todas las muestras no-inoculadas, la evaluación de E. coli, Listeria spp. y Salmonella spp. arrojó resultados negativos (ausencia del patógeno). Con estos resultados se comprobó que los recuentos de los patógenos en las salchichas y mortadelas inoculadas se debían únicamente a la inoculación. Los valores promedio para el pH y Aw de ambos productos permaneció invariable durante el estudio, con valores dentro del rango 5,9-6,2 y alrededor de 0,977 ; respectivamente. El promedio del $\mathrm{pH}$ y el Aw al inicio del estudio fue similar en todos los tratamientos. Al inicio del estudio el recuento de microorganismos aerobios mesófilos de los productos eran muy similares entre sí (2,5-4,0 Log UFC/g), pero en la mitad de la vida útil (semana 3), el promedio de los productos elaborados con lactato de sodio $2,5 \%$ y los que no llevaban ningún antimicrobiano (Control), superaron el límite máximo permitido para estos productos (> 5,0 Log UFC/g) puesto que alcanzaron recuentos superiores a $12 \mathrm{Log}$ UFC/g, mientras que los productos con el antimicrobiano natural en cualquiera de la dosis estudiada mantuvo el promedio de los recuentos de microorganismos aerobios por debajo de $5 \mathrm{Log}$ UFC/g hasta la tercera semana (Tabla 2). En la Tabla 2, se muestra el promedio de Log UFC/g con su respectiva desviación estándar para tres mediciones y tres réplicas. Los subíndice $i$ y $f$ indican el valor inicial y final de cada parámetro, respectivamente. Letras iguales en cada columna, indican que no existe diferencias estadísticamente significativas entre los tratamientos $(p<0.05)$.

Tabla 2: Evaluación del pH, Aw y microorganismos aerobios mesófilos en los productos no-inoculados.

\begin{tabular}{|c|c|c|c|c|c|}
\hline $\begin{array}{c}\text { Producto/ } \\
\text { Tratamiento Antimicrobiano }\end{array}$ & $\mathrm{pH}_{i}$ & $A w_{i}$ & $\begin{array}{l}\log ^{*} \\
(U F C / g)_{i}\end{array}$ & $\begin{array}{c}\text { Log }^{*} \text { UFC } / g \\
\text { semana } 3\end{array}$ & $\log ^{*}(U F C / g)_{f}$ \\
\hline Salchicha & & & & & \\
\hline Control (sin antimicrobiano) & $5,80 \pm 0,05^{a}$ & $0,986 \pm 0,01^{b}$ & $3,6 \pm 1,0^{c}$ & $7,75 \pm 0,75^{d}$ & $>12,0^{9}$ \\
\hline Antimicrobiano natural $(0,3 \%)$ & $5,95 \pm 0,01^{a}$ & $0,980 \pm 0,02^{b}$ & $3,5 \pm 0,7^{c}$ & $4,00 \pm 0,5^{\mathrm{e}}$ & $9,0 \pm 0,5^{\mathrm{h}}$ \\
\hline Antimicrobiano natural $(0,65 \%)$ & $6,05 \pm$ & $0,97 \varepsilon$ & $3,7 \pm 0,9^{c}$ & $3,75 \pm 0,55^{\mathrm{e}}$ & $8,05 \pm 0,55^{h}$ \\
\hline $\begin{array}{l}\text { Lactato de sodio }(2,5 \%) \\
\text { Mortadela }\end{array}$ & $5,88 \pm 0,06^{a}$ & $0,980 \pm 0,02^{b}$ & $3,5 \pm 1,1^{c}$ & $7,95 \pm 0,45^{d}$ & $>12,0^{9}$ \\
\hline Control (sin antimicrobiano) & $5,86 \pm 0,04^{a}$ & $0,978 \pm 0,05^{b}$ & $3,5 \pm 0,8^{c}$ & $8,2 \pm 0,70^{d}$ & $>12,0^{9}$ \\
\hline Antimicrobiano natural $(0,3 \%)$ & & $0,980 \pm 0$, & $3,7 \pm 0,9^{c}$ & $4,15 \pm 0,45^{\mathrm{e}}$ & $9,3 \pm 0,4^{\mathrm{h}}$ \\
\hline Antimicrobiano natural $(0,65 \%)$ & $5,95 \pm 0,02^{\mathrm{a}}$ & $0,979 \pm 0,01^{b}$ & $3,4 \pm 1,0^{c}$ & $3,9 \pm 0,50^{\mathrm{e}}$ & $7,4 \pm 0,9^{\mathrm{h}}$ \\
\hline Lactato de sodio $(2,5 \%)$ & $5,85 \pm 0,07^{a}$ & $0,979 \pm 0,02^{b}$ & $3,6 \pm 1,2^{c}$ & $8,35 \pm 0,85^{d}$ & $>12,0^{g}$ \\
\hline
\end{tabular}

Tabla 3: Modelos matemáticos para el crecimiento de E. coli, L. monocytogenes y S. Typhimurium en los productos inoculados

\begin{tabular}{|c|c|c|c|c|c|}
\hline \multirow{2}{*}{ Microorganismo } & \multirow{2}{*}{ Tratamiento } & \multicolumn{4}{|c|}{ Coeficientes } \\
\hline & & $\beta_{0}$ & $\beta_{1}$ & $\beta_{2}$ & $\beta_{3}$ \\
\hline \multirow{4}{*}{$\begin{array}{c}\text { E. coli } \\
\text { Log UFC/g }=\beta_{0}+\beta_{1} T+\beta_{2} T^{2}+\beta_{3} T^{3}\end{array}$} & Antimicrobiano natural $(0,3 \%)$ & 1,143 & $-0,469$ & 0,026 & 0,004 \\
\hline & Antimicrobiano natural $(0,65 \%)$ & 0,934 & $-0,238$ & $-0,577$ & $-0,021$ \\
\hline & Lactato de sodio $(2,5 \%)$ & 0,934 & $-0,6831$ & 0,176 & $-0,015$ \\
\hline & Control (sin antimicrobiano) & 0,958 & 0,175 & $-0,164$ & 0,018 \\
\hline \multirow{4}{*}{$\begin{array}{c}\text { L. monocytogenes } \\
\text { Log UFC/g }=\beta_{0}+\beta_{1} T+\beta_{2} T^{2}\end{array}$} & Antimicrobiano natural $(0,3 \%)$ & 1,399 & $-0,578$ & 0,053 & - \\
\hline & Antimicrobiano natural $(0,65 \%)$ & 1,422 & $-0,578$ & 0,053 & - \\
\hline & Lactato de sodio $(2,5 \%)$ & 1,541 & $-0,578$ & 0,053 & - \\
\hline & Control (sin antimicrobiano) & 2,160 & $-0,578$ & 0,053 & - \\
\hline \multirow{4}{*}{$\begin{array}{c}\text { S. Typhimurium } \\
\text { Log UFC/g }=\beta_{0}+\beta_{1} T+\beta_{2} T^{2}+\beta_{3} T^{3}\end{array}$} & Antimicrobiano natural $(0,3 \%)$ & 1,734 & $-0,229$ & $-0,091$ & 0,022 \\
\hline & Antimicrobiano natural & 1,754 & 0,646 & $-0,297$ & 0,022 \\
\hline & Lactato de sodio $(2,5 \%)$ & 1,662 & 1,225 & $-0,395$ & 0,022 \\
\hline & Control (sin antimicrobiano) & 1,610 & 1,803 & $-0,43$ & 0,022 \\
\hline
\end{tabular}




\section{Crecimiento de E. coli, L. monocytogenes y S. Typhimurium}

Los modelos que mejor se ajustan a los datos de Log UFC/g para E. coli y S. Typhimurium tienen forma cúbica, mientras que para L. monocytogenes se observó un mejor ajuste con ecuaciones de tipo cuadrático. El crecimiento de estos microorganismos durante el estudio, se observa en la Figura 2. En estos modelos el tiempo $(T)$ en semanas y los tratamientos son los efectos fijos que ayudan a estimar el recuento de cada microrganismo. Los coeficientes de cada ecuación $\left(\beta_{0}, \beta_{1}, \beta_{2}\right.$ y $\left.\beta_{3}\right)$ se presentan en la Tabla 3.
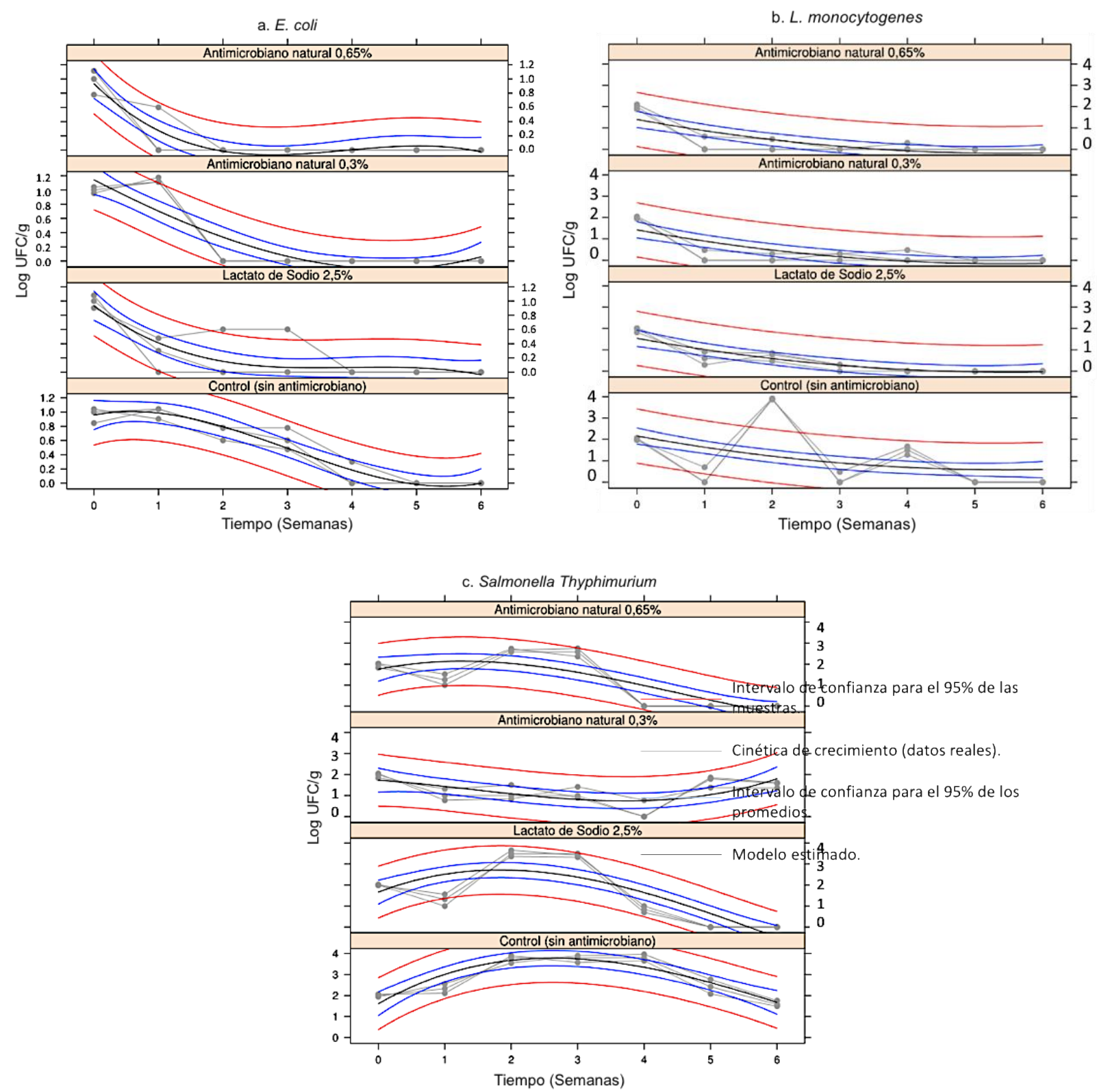

Fig. 2: Crecimiento de a. E. coli; b. L. monocytogenes y c. S. Typhimurium en los productos inoculados con cada microorganismo.

Porcentaje de inhibición de E. coli, L. monocytogenes y S. Typhimurium

El cálculo del porcentaje de inhibición de E. coli, L. monocytogenes y S. Typhimurium con cada uno de los antimicrobianos (Tabla 4), se obtuvo comparando el crecimiento del microrganismo sin ninguna sustancia antimicrobiana (Control) y el obtenido en el mismo tiempo con el antimicrobiano en cuestión, usando la ecuación (1).

$\%$ Inhibición $=\frac{\left(\log \frac{\mathrm{UFC}}{\mathrm{g}} \text { control }-\log \frac{\mathrm{UFC}}{\mathrm{g}} \text { antimicrobiano }\right)}{\left(\log \frac{\mathrm{UFC}}{\mathrm{g}} \text { control }\right)} \times 100 \%$ 
En la Tabla 4, las letras iguales indican que no existe diferencia estadísticamente significativa $(p<0.05)$ entre los promedios del Log UFC/g de tres muestreos y tres réplicas, para cada tratamiento en cada semana, correspondientes al $95 \%$ de las muestras. En algunos casos, el recuento de microorganismo es cero también en el control y se representa como na.

Tabla 4: Porcentaje de inhibición de E. coli, L. monocytogenes y S. Typhimurium.

\begin{tabular}{|c|c|c|c|c|c|c|c|}
\hline \multirow[b]{2}{*}{$\begin{array}{l}\text { Microorganismo } \\
\text { inoculado }\end{array}$} & \multirow[b]{2}{*}{ Tratamiento } & \multicolumn{6}{|c|}{ \% Inhibición ${ }^{* *}$} \\
\hline & & $\begin{array}{c}\text { Semana } \\
1\end{array}$ & $\begin{array}{l}\text { Semana } \\
2\end{array}$ & $\begin{array}{c}\text { Semana } \\
3\end{array}$ & $\begin{array}{c}\text { Semana } \\
4\end{array}$ & $\begin{array}{c}\text { Semana } \\
5\end{array}$ & $\begin{array}{c}\text { Semana } \\
6\end{array}$ \\
\hline \multirow{3}{*}{ E. coli } & Antimicrobiano natural $(0,3 \%)$ & $35,0^{a}$ & $70,0^{\mathrm{a}}$ & $100,0^{\mathrm{a}}$ & $100,0^{\mathrm{a}}$ & na & na \\
\hline & $\begin{array}{l}\text { Antimicrobiano natural }(0,65 \\
\%)\end{array}$ & $72,0^{\mathrm{b}}$ & $100,0^{\mathrm{a}}$ & $100,0^{\mathrm{a}}$ & $100,0^{a}$ & na & na \\
\hline & Lactato de sodio (2,5 \%) & $60,0^{c}$ & $33,3^{a}$ & $88,9^{a}$ & $100,0^{a}$ & na & na \\
\hline \multirow{3}{*}{$\begin{array}{c}L . \\
\text { monocytogenes }\end{array}$} & Antimicrobiano natural $(0,3 \%)$ & $41,2^{\mathrm{a}}$ & $66,7^{a}$ & $90,0^{a}$ & $86,7^{a}$ & $100,0^{\mathrm{a}}$ & $100,0^{a}$ \\
\hline & $\begin{array}{l}\text { Antimicrobiano natural }(0,65 \\
\%)\end{array}$ & $47,1^{\mathrm{a}}$ & $58,3^{a}$ & $100,0^{\mathrm{a}}$ & $100,0^{\mathrm{a}}$ & $100,0^{a}$ & $100,0^{\mathrm{a}}$ \\
\hline & Lactato de sodio (2,5 \%) & $41,2^{a}$ & $41,7^{a}$ & $80,0^{\mathrm{a}}$ & $100,0^{a}$ & $100,0^{a}$ & $100,0^{\mathrm{a}}$ \\
\hline \multirow{3}{*}{ S. Typhimurium } & Antimicrobiano natural $(0,3 \%)$ & $85,0^{a}$ & $71,6^{a}$ & $76,0^{\mathrm{a}}$ & $75,8^{a}$ & $59,6^{a}$ & $-9,1^{b}$ \\
\hline & $\begin{array}{l}\text { Antimicrobiano natural }(0,65 \\
\%)\end{array}$ & $31,7^{\mathrm{a}}$ & $45,9^{a}$ & $58,7^{b}$ & $69,7^{a}$ & $88,5^{a}$ & $100,0^{a}$ \\
\hline & Lactato de sodio (2,5 \%) & $13,3^{a}$ & $52,7^{a}$ & $34,7^{b}$ & $47,0^{b}$ & $73,1^{a}$ & $100,0^{a}$ \\
\hline
\end{tabular}

\section{DISCUSIÓN}

Aunque las bacterias Gram-negativas son generalmente más resistentes a los antimicrobianos de origen vegetal e incluso puede que no se observe ningún efecto, en comparación con las bacterias Gram-positivas (Biswas et al., 2013), para E. coli se observó inhibición mayor al 50 \% respecto al control sin antimicrobiano desde la primera semana en los productos con $2,5 \%$ de lactato de sodio y $0,65 \%$ de antimicrobiano natural, y se redujo su población hasta en un $100 \%$ a partir de la segunda semana para la concentración más alta del antimicrobiano natural, y en la tercera semana para los productos con $2.5 \%$ de lactato de sodio. Durante la fermentación y el almacenamiento a $4-30{ }^{\circ} \mathrm{C}$ de salchichas maduradas y semi-maduradas, también se ha observado mayor reducción de la población de E. coli que de L. monocytogenes (Dalzini et al., 2015; Valimaa et al., 2015). En este estudio se observó una reducción en el crecimiento de $E$. coli en todas las muestras, incluso en las muestras del tratamiento control (sin antimicrobiano) a partir de la semana cinco, lo que podría explicarse por el efecto "Jameson" (Jameson, 1962; Condón et al., 2016) en el que se suprime el crecimiento de la población que se encuentra en menor proporción ( $E$. coli) respecto de otras poblaciones presentes (aerobios mesófilos). Este efecto se ha descrito también al observar el crecimiento simultáneo de $L$. monocytogenes y las bacterias ácido lácticas (BAL) en el queso cottage (Bjerre et al., 2014).

Los modelos matemáticos cuadráticos sirvieron para estimar el crecimiento E. coli y S. Typhimurium; mientras que un modelo cúbico fue más útil para describir el comportamiento de L. monocytogenes debido a la forma cóncava en todos los tratamientos. El empleo de antimicrobiano natural con extracto de romero en las dos concentraciones estudiadas logró en la segunda semana, una reducción mayor al 50 \% de L. monocytogenes, mientras que para este mismo período, el lactato de sodio solo logró reducir el 41,7\% de la población del microorganismo. A partir de la tercera semana, la inhibición de este patógeno fue mayor al $80 \%$ respecto al control sin antimicrobiano. Debido a la limitada acción antimicrobiana del lactato de sodio frente a patógenos como L. monocytogenes, se han realizado numerosos estudios que han mostrado un efecto sinérgico entre el lactato (1,5\%- 3,0 \%) y el diacetato de sodio $(0,125 \%-0,25 \%)$ sobre la inhibición del crecimiento de microorganismos patógenos (Dalzini et al., 2015).y en productos de carne y aves de corral (Hwang et al., 2015).

Es posible que la baja eficacia de los antimicrobianos estudiados sobre el crecimiento de $S$. Typhimurium se deba a una alta tasa de recuperación de las células que se encontraban con daño sub-letal (Hrabak et al., 2015) en el medio de enriquecimiento usado para realizar los recuentos del microorganismo. Se debe considerar también, que en efecto $S$. Typhimurium es menos sensible a los antimicrobianos empleados y por esto sea recomendable emplear otros antimicrobianos y tecnologías que ayuden a controlar el crecimiento de Salmonella spp. en derivados cárnicos. Mbandi y Shelef (2002) reportaron la inhibición de Salmonella serotipo Enteritidis mediante la adición de una mezcla de lactato de sodio $(2,5 \%)$ y diacetato de sodio $(0,2 \%)$ en carne 
conservada a baja temperatura $\left(5\right.$ y $\left.10^{\circ} \mathrm{C}\right)$. Los mismos investigadores probaron que la combinación de lactato de sodio con diacetato, incluso ayuda a controlar el crecimiento de los microorganismos aerobios en el producto, y por tanto, prolonga su vida útil después de que se abren los empaques. De igual manera, Miladi et al. (2016) sugieren que el aceite esencial de $R$. officinalis podría inhibir in vitro la formación de biopelículas de varias cepas de Salmonella pero en concentraciones altas (>200 mg/mL) (Miladi et al., 2016); sin embargo, en este caso emplear una concentración mayor del antimicrobiano natural para garantizar la inhibición de esta bacteria no sería posible, debido a que afectaría negativamente las características sensoriales de los productos.

En general, se esperaba un mayor efecto inhibitorio de ambos antimicrobianos sobre los microorganismos estudiados, basado en los valores de CMI previamente encontrados para este estudio (datos no mostrados); lo cual valida lo reportado por Gutierrez et al. (2008) quienes sugieren que si bien se ha demostrado in vitro que los extractos de plantas son útiles para la reducción de patógenos asociados con productos cárnicos, el efecto antimicrobiano en los productos es menor debido al efecto antagónico producido por la grasa presente en la fórmula (Gutierrez et al., 2008). Los valores de $\mathrm{pH}$ y Aw de las mortadelas y salchichas que no se inocularon, no mostraron diferencias estadísticamente significativas $(p>0,05)$ entre los tratamientos durante la vida útil de los productos (seis semanas). Sin embargo, se observó un aumento en el recuento de microorganismos aerobios mesòfilos en los productos sin ningún antimicrobiano (Control) y lactato de sodio, a partir de la tercera semana.

Aunque en los últimos años se ha estudiado ampliamente la actividad antimicrobiana in vitro de un gran número de compuestos naturales entre los que se encuentran los aceites esenciales, son menos los estudios que se han realizado en matrices alimenticias reales para evaluar el efecto de la adición de estos compuestos sobre las características sensoriales del producto y la interacción con los demás ingredientes de la fórmula. (Viuda et al., 2010). En general, el uso de plantas con un alto contenido de timol, carvacrol, linalol, 1, 8-cineol, $\alpha$-pineno o a-terpineol, solos o en combinación en los alimentos, debería analizarse con detenimiento; pues el efecto antimicrobiano deseado de estas sustancias se produce a concentraciones inaceptables desde el punto de vista sensorial (Klein et al., 2013).

\section{CONCLUSIONES}

En los productos empacados al vacío, refrigerados $\left(4-5^{\circ} \mathrm{C}\right)$, e inoculados con una concentración inicial de $L$. monocytogenes de $10^{2}-10^{3} \mathrm{UFC} / \mathrm{mL}$; sólo el antimicrobiano natural al $0,65 \%$ inhibió por completo el crecimiento de este patógeno al alcanzar la tercera semana. En el caso de $S$. Typhimurium, ninguno de los antimicrobianos estudiados logró inhibir completamente al microorganismo; únicamente se observó el $100 \%$ de la inhibición al final del estudio en los productos que contenían la mayor concentración de antimicrobianos $(0,65 \%$ de antimicrobiano natural y $2,5 \%$ lactato de sodio). En salchicha fue posible observar el efecto inhibitorio completo de $E$. coli con $0,65 \%$ del antimicrobiano natural a partir de la segunda semana. En general, los derivados cárnicos cuya fórmula contienen algún antimicrobiano inhiben el crecimiento de los microorganismos estudiados, pero su eficacia es mayor frente a E. coli y L. monocytogenes, y muy limitada frente a $S$. Typhimurium.

\section{AGRADECIMIENTOS}

A la empresa Tecnas S.A. (Itagüí, Colombia) por el apoyo técnico y financiero brindado para el desarrollo de este trabajo.

\section{REFERENCIAS}

Ahmed, O. M., P. Pangloli y otros ocho autores, The Occurrence of Listeria monocytogenes in Retail Ready-to-Eat Meat and Poultry Products Related to the Levels of Acetate and Lactate in the Products, Food Control, 52,43e48 (2015)

Bezic, N., V. Dunkic y E. Vuko, Antiphytoviral Activity of Essential Oils of Some Lamiaceae Species and There Most Important Compounds on CMV and TMV In: Microbial pathogens and strategies for combating them: science, technology and education, Ed.: A. Méndez-Vilas, Formatex Research Center, Badajoz, 2, 982-988 (2013)

Biswas, B., K. Rogers y otros tres autores, Antimicrobial Activities of Leaf Extracts of Guava (Psidium guajava L.) on Two Gram-Negative and Gram-Positive Bacteria, International Journal of Microbiology, 2013 (1), 1-7 (2013)

Bjerre, N., A. Eklöw y P. Dalgaard, Modelling the Effect Of Lactic Acid Bacteria From Starter- and Aroma Culture on Growth of Listeria monocytogenes in Cottage Cheese, doi:10.1016/j.ijfoodmicro.2014.07.012, International Journal of Food Microbiology, 188 (October), 15-25 (2014)

Bubonja, M., J. Giacometti y M. Abram, Antioxidant and Antilisterial Activity of Olive Oil, Cocoa and Rosemary Extract Polyphenols, Food Chemistry, 127 (4), 1821-1827 (2011) 
Condón, S., J. Raso y otros dos autores, Inactivation of Salmonella typhimurium and Lactobacillus plantarum by UV-C Light in Flour Powder, Innovative Food Science and Emerging Technologies, 35, 1-8 (2016)

Dalzini, E., E. Cosciani y otros cinco autores, Behaviour of Escherichia coli O157 (VTEC), Salmonella Typhimurium and Listeria monocytogenes During the Manufacture, Ripening and Shelf Life of Low Fat Salami, Food Control, 47, 306-311 (2015)

Gutierrez, J., C. Barry y P. Bourke, The Antimicrobial Efficacy of Plant Essential Oil Combinations and Interactions with Food Ingredients, International Journal of Food Microbiology, 124 (1), 91-97 (2008)

Ghabraie, M., K. Dang y otros tres autores, Antimicrobial Effect Of Essential Oils in Combinations Against Five Bacteria and Their Effect on Sensorial Quality of Ground Meat, LWT - Food Science and Technology, 66, 332-339 (2016)

Hrabak, J., E. Chud y C. Papagiannitsis, Detection of Carbapenemases in Enterobacteriaceae: A Challenge for Diagnostic Microbiological Laboratories, Clinical Microbiology and Infection, 20 (9), 839-853 (2014)

Hwang, C., Li. Huang y V. Juneja, Effect of Acidified Sorbate Solutions on the Lag-Phase Durations and Growth Rates of Listeria monocytogenes on Meat Surfaces, Journal of Food Protection, 6, 1064-1243 (2015)

Instituto Colombiano de Normas Técnicas y Certificación, Norma Técnica Colombiana. NTC 4519. Microbiología de los Alimentos para Consumo Humano y Animal. Método Horizontal para el Recuento de Microorganismos, Técnica de Recuento de Colonias a $30^{\circ} \mathrm{C}$. Primera actualización, ICONTEC, Bogotá, p. 9 (2009a)

Instituto Colombiano de Normas Técnicas y Certificación, Norma Técnica Colombiana. NTC 4516. Microbiología de Alimentos y Productos de Alimentación Animal. Método Horizontal para la Detección y Enumeración de Coliformes Técnica del Número Más Probable. Primera actualización, ICONTEC, Bogotá, p. 11 (2009b)

Instituto Nacional de Vigilancia de Medicamentos y Alimentos, INVIMA, https://www.invima.gov.co/principal.htm, Colombia (2018)

Jameson, J.E., A discussion of the Dynamics of Salmonella enrichment, J. Hyg., 60 (2), 193-207 (1962)

Kalschne, D., R. Womer y otros cuatro autores, Characterization of the Spoilage Lactic Acid Bacteria in "Sliced VacuumPacked Cooked Ham", Brazilian Journal of Microbiology, 46(1), 173-181 (2015)

Klein, G., C. Rüben y M. Upmann, Antimicrobial Activity of Essential Oil Components against Potential Food Spoilage Microorganisms, Curr. Microbiol, Journal of Food Microbiology, 45, 43-53 (2013)

Littell, R., G. Milleken y otros tres autores, SAS for Mixed Models, $2^{\text {nd }}$ Ed., Institute Inc., Cary, EE.UU. p. 814 (2006)

Miladi, H., D. Mili y otros cuatro autores, Antibiofilm Formation and Anti-adhesive Property of Three Mediterranean Essential Oils against a Foodborne Pathogen Salmonella Strain, doi:10.1016/j.miicpath.2016.01.017, Microbial Pathogenesis, 93(April), 22-31 (2016)

Mbandi, E. y L. A. Shelef, Enhanced Inhibition of Listeria monocytogenes and Salmonella enteritidis in Meat by Combinations of Sodium Lactate and Diacetate, Journal of Food Protection, 64(5), 640 (2001)

Muriel, V., M. Cran y otros tres autores, Antioxidant and Antimicrobial Properties of Ethylene Vinyl Alcohol Copolymer Films Based on the Release of Oregano Essential Oil and Green Tea Extract Components, J. Foor Eng., 149, 9-16 (2015)

National Center for Emerging \& Zoonotic Infectious Diseases, Division of Foodborne, Waterborne, and Environmental Diseases, Trends in Foodborne Illness, 1996-2010 (2011)

Organización Internacional de Normalización, ISO 11290-2, "Microbiology of Food and Animal Feeding Stuffs - Horizontal Method for The Detection and Enumeration of Listeria monocytogenes - Part 2: Enumeration method" (1998)

Organización Internacional de Normalización, ISO 6579, Microbiología de los Alimentos Para Consumo Humano y Alimentación Animal, Método horizontal Para la Detección de Salmonella spp. 2003

Pereira, C., E. Colla y otros tres autores, Food Additives Reduce Lactic Acid Bacterial Growth in Culture Medium and in Meat Products, Increasing Product Shelf Life, Semina: Ciências Agrárias, Londrina, 36(6), 3681-3698 (2015)

Pisoschi, A.M., A. Pop y otros cuatro autores, An Overview of Natural Antimicrobials Role in Food, doi: 10.1016/j.ejmech.2017.11.095, European Journal of Medicinal Chemistry, 143, 922-935 (2018)

Posada S. y R. Rosero, Comparación de Modelos Matemáticos: Una Aplicación en la Evaluación de Alimentos para Animales. Rev. Col. Cienc. Pec., 20, 141 (2007)

R CORE TEAM. R, A language and environment for statistical computing, ISBN: 3-900051-07-0. R Foundation for Statistical Computing, Vienna, Austria (2012)

Singh, A., P.K. Sharma y G. Garg, Natural Products as Preservatives, Int. J. Pharma Bio Sci., 1, 601- 612 (2010)

Valimaa, A., A. Timisjarvi y E. Virtanen, Rapid detection and identification methods for Listeria monocytogenes in the food chain - A review, Food Control, 55, 103-114 (2015)

Viuda, M., Y. Ruiz y otros dos autores, Effect of added citrus fibre and spice essential oils on quality characteristics and shelf-life of mortadella, Meat Science, 85 (3), 568-576 (2010) 
